\title{
Monitorage du tarif ambulatoire fondé sur les preuves
}

\author{
Kerstin Schutz, Sabine Zehnder
}

Expertes, division Médecine et tarifs ambulatoires

\begin{abstract}
Si plusieurs acteurs discutent de l'évolution de coûts de la santé, ils les interprètent différemment. Grâce à la collecte de données par et pour les médecins, la FMH est en mesure d'analyser les évolutions du secteur ambulatoire et de les placer dans un contexte médical.
\end{abstract}

L’agenda politique est marqué depuis des années par l'évolution des coûts de la santé et une des missions de la FMH, en sa qualité de représentante des médecins en Suisse, consiste à suivre et à expliquer les évolutions. Pour le secteur ambulatoire, l'évolution des coûts peut avoir différentes causes, sans être obligatoirement liée à une augmentation du volume induite par les médecins.

Ces trois dernières années, pas moins de 10000 cabinets médicaux ont livré activement leurs données.

La collecte des données par et pour les médecins est au cœur du monitorage tarifaire de la FMH. Ces trois dernières années, pas moins de 10000 cabinets médicaux ont livré activement leurs données et financé cette collecte par leurs cotisations aux centres de confiance cantonaux. Vu que la Société vaudoise de médecine (SVM) et des parties de Suisse centrale poursuivent leurs propres solutions, nous atteignons une couverture de près de $65 \%$ sur l'ensemble de la Suisse. Le pool de données du corps médical suisse comprend actuellement 275 millions de factures (copies), soit près de 2,8 milliards de fichiers et de données de prestations (état: mi-juin 2019). De cette manière, les données peuvent être analysées selon les indications disponibles sur chaque facture. Ces données sont préparées par NewIndex/TrustX et mises à la disposition de la FMH sous forme anonymisée. La protection des données est toujours garantie et le codex $\mathrm{NAKO}^{1}$ strictement respecté.

Pour ses analyses, la FMH observe les évolutions «par unité» relative, c'est-à-dire par exemple par patient, par cabinet ou par séance. Cela permet de négliger les effets exogènes lors de l'analyse des causes possibles et les données analysées sont plus précises qu'un pour- centage indiquant l'augmentation de l'ensemble des coûts. Il est bien évident que ces chiffres sont importants; il faut considérer les coûts dans leur ensemble ou sur la base du volume général de points tarifaires mais ces valeurs ne constituent qu'un aspect de la vue générale et, par ailleurs, elles ne tiennent pas compte de nombreux facteurs exogènes comme les évolutions démographiques ou les décisions politiques telles que privilégier l'ambulatoire à l'hospitalier.

Les valeurs clés suivantes sont analysées et contextualisées (entre autres):

- Volume par séance (TARMED)

Il s'agit des coûts (nombre de points tarifaires) d'une séance moyenne. Pour une meilleure vue d'ensemble, les positions tarifaires sont classées en différents blocs, p. ex. les prestations appartenant typiquement à une consultation de base. Ce type d'analyses permet d'avoir un point de vue nuancé sur le changement de volume des positions tarifaires et le met en relation avec d'autres évolutions. De cette manière, il devient possible de relativiser les effets pouvant apparaître comme «inquiétants» alors qu'ils ne représentent qu'une part marginale du volume général et, inversement, de mettre en évidence l'influence que peuvent avoir des changements apparemment mineurs sur le volume général.

- Volume par patient (TARMED)

Le volume par patient indique les coûts (nombre de points tarifaires) par patient facturés avec le TARMED par un médecin pendant une période précise.

- Volume par cabinet (TARMED)

Le volume par cabinet correspond aux coûts moyens (nombre de points tarifaires) d'un cabinet médical pendant une période donnée. Un cabinet médical correspond à un numéro RCC. 


\section{- Séances par patient}

Le nombre de séances par patient indique le nombre de fois qu'un patient est allé consulter son médecin pendant une période donnée. Une séance se définit par un contact par jour avec le patient.

- Séances par cabinet médical

Le nombre de séances par cabinet médical comprend le nombre de séances de tous les patients d'un cabinet pendant une période donnée. Un cabinet médical correspond à un numéro RCC.

\section{- Patients par cabinet médical}

Le nombre de patients par cabinet médical indique combien de patients (premiers contacts) ont été traités pendant une période donnée par un médecin. Un cabinet médical correspond à un numéro RCC.

\section{Contextualisation}

Cette dimension permet d'analyser différents effets: Le graphique ci-après représente par exemple la hausse de toutes les séances depuis 2016. Cette évolution est cependant relativisée par le nombre de patients (premiers contacts), qui a également augmenté par cabinet.

Au cours des six dernières années, le nombre de séances par patient a connu une hausse moyenne de $0,64 \%$ par an.

Le nombre de séances par patient a augmenté de manière marginale en 2019 (+0,62\% par rapport à l'année précédente). Au cours des six dernières années, cette hausse a été en moyenne de 0,64\% par an. La hausse actuelle est donc dans la moyenne des dernières années et les médecins n'ont pas forcément fait venir leurs patients plus souvent qu'avant l'intervention tarifaire de 2018.

Le volume de points du TARMED par séance a continué de baisser en 2019 de -0,26\% (2018: -3,69\%).
Le volume de points du TARMED par patient indique en 2019 une augmentation inférieure à la moyenne $(0,38 \%)$ par rapport à l'année précédente. La hausse moyenne de ces six dernières années ayant été de 1,71\% par patient.

Afin de pouvoir comparer des analyses, il est indispensable d'en connaître les définitions et la base des données.

\section{Comparabilité: date de traitement médi- cal et date de traitement administratif}

Prenons l'exemple du monitoring de l'évolution des coûts de l'assurance-maladie (MOKKE) réalisé par l'Office fédéral de la santé publique (OFSP) qui, lui aussi, évalue les coûts par unité, mais dans son cas, l'unité représente les coûts globaux par personne assurée, tous secteurs et fournisseurs de prestations confondus. Ce monitorage se fonde sur les données de l'assurance-maladie et, plus exactement, sur les données datées du jour où la prestation a été traitée au sein de l'assurance-maladie (date de traitement administratif).

En revanche, les analyses de la FMH reposent sur la date du traitement médical, à savoir le jour où la prestation a été fournie. Cela permet de passer à la loupe les effets saisonniers ou les événements extraordinaires. Si ces deux méthodes se complètent, elles se distinguent en raison du fait que la FMH met l'accent sur l'économie d'entreprise des cabinets médicaux tandis que MOKKE se concentre plutôt sur les aspects d'économie politique/macroéconomiques.

\section{Comparabilité: personne malade et personne assurée}

Les notions de "personne assurée» (MOKKE) et de "personne malade» (monitorage de la FMH) sont un autre

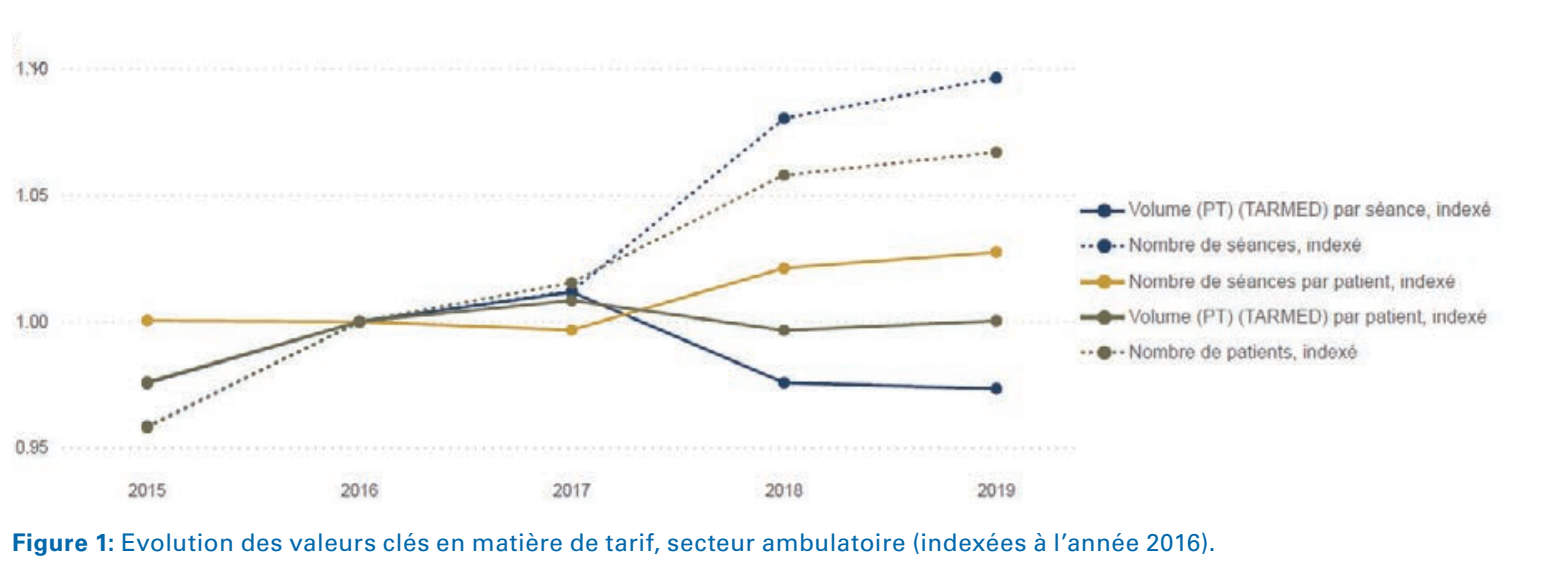


FMH/division Médecine et tarifs ambulatoires

Baslerstrasse 47 CH-4600 Olten

Tél. 0313591230

Fax 0313591238

tarife.ambulant[at]fmh.ch exemple rappelant qu'il faut être prudent lors de comparaison avec les données MOKKE. Les coûts par personne assurée représentent la somme de tous les traitements ambulatoires prodigués (TARMED, forfaits négociés, etc.) par plusieurs fournisseurs de prestations. Inversement, le volume de points tarifaires par patient (personne malade) du monitorage de la FMH ajoute la distinction du volume par fournisseur de prestations. Par exemple, pour un patient qui se rend chez son médecin de famille et chez un spécialiste pendant une période donnée, le monitorage de la FMH sépare les coûts en fonction du médecin (numéro $\mathrm{RCC}$ ) et la personne concernée équivaut alors à deux personnes malades pendant cette période.

\section{Observation différenciée et autres mesures de référence}

Les données collectées par les médecins permettent de porter un regard différencié sur l'évolution des données de facturation dans le secteur ambulatoire.

$\mathrm{Au}$-delà des analyses en fonction des indicateurs présentés ici, beaucoup d'autres analyses sont aussi réalisées: entre autres sur l'évolution de la séance moyenne et de sa composition ou encore sur les prestations fournies conjointement lors d'une séance (co-positions). Il est par ailleurs possible de procéder à des analyses dif- férenciées pour des groupes de patients déterminés (p. ex. en fonction de l'âge ou du sexe). Enfin, les analyses concernant le tarif médical TARMED sont complétées par les analyses du volume de facturation de la liste des analyses (laboratoire du praticien) ou de médicaments.

Ces analyses complètes sont uniquement possible grâce à tous les cabinets médicaux qui transmettent leurs données de facturation.

Il est bien évident que la FMH se réfère à d'autres publications pour ses analyses.

Ces analyses complètes sont uniquement possibles grâce à tous les cabinets médicaux qui transmettent leurs données de facturation aux centres de confiance cantonaux. Leur nombre ne cesse de s'accroître et nous remercions vivement tous les médecins qui fournissent leurs données, car ils alimentent notre réservoir de données, le financent et contribuent ainsi à ce que la FMH puisse fournir des analyses précises de l'activité du corps médical.

Nous adressons également nos plus vifs remerciements à la Caisse des médecins pour son énorme savoir-faire qui nous aide et nous conseille activement lors de la préparation et de la réalisation de ce monitorage.

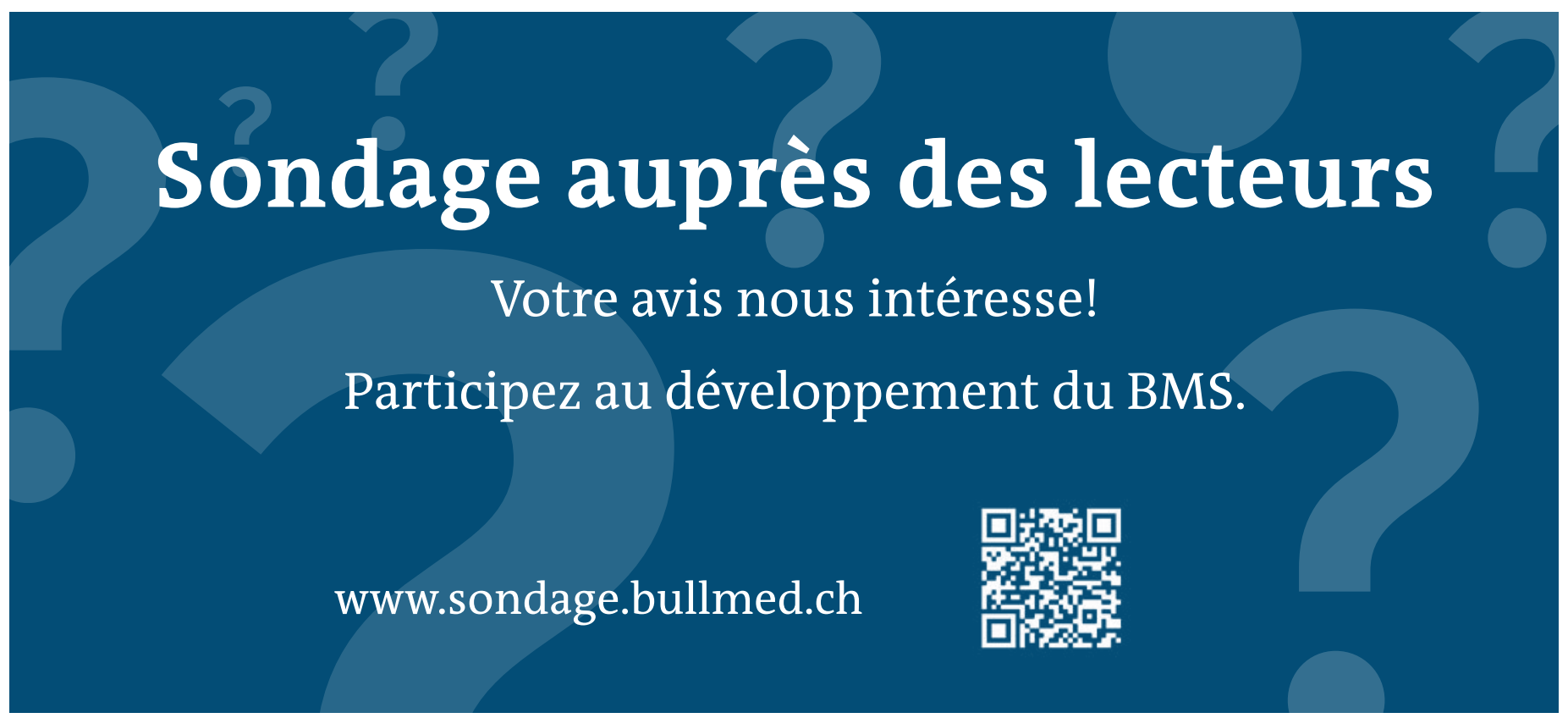

